International Mathematical Forum, Vol. 9, 2014, no. 24, 1177 - 1190

HIKARI Ltd, www.m-hikari.com

http://dx.doi.org/10.12988/imf.2014.45108

\title{
An Efficient Technique for Solving Gas Dynamics Equation Using the Natural Decomposition Method
}

\author{
Shehu Maitama \\ Department of Mathematics, Northwest University, Kano \\ Kabuga-Katsina Road P.M.B. 3099 Kano State, Nigeria \\ Sabuwa Mustapha Kurawa \\ Department of Physics, Sa'adatu Rimi College of Education Kumbotso, Kano \\ Zaria Road 3218, Nigeria \\ Copyright (c) 2014 Shehu Maitama and Sabuwa Mustapha Kurawa. This is an open \\ access article distributed under the Creative Commons Attribution License, which permits \\ unrestricted use, distribution, and reproduction in any medium, provided the original work \\ is properly cited.
}

\begin{abstract}
In this paper, we introduce a new technique called the Natural Decomposition Method (NDM) for solving homogeneous and inhomogeneous gas dynamic equations. The new method is an elegant combination of the Natural transform Method (NTM) and Adomian Decomposition Method (ADM). The proposed method is applied directly without using linearization, transformation, discretization or taking some restrictive assumptions. Using the new method, we successfully obtain exact solution of two illustrative examples, and the results are compared with the results of the existing methods. This shows the reliability, accuracy, and efficiency of the new method.
\end{abstract}

Mathematics Subject Classification: 35Q51, 74G15

Keywords: Natural Decomposition Method, Adomian polynomials, Nonlinear Gas Dynamics Equations 


\section{Introduction}

Gas dynamic equations are the mathematical expressions of conservation laws which exist in physical and applied science practices such as conservation of momentum, conservation of mass, conservation of energy and so on. The standard gas dynamic equation in one spacial dimension is given by:

$$
v_{t}(x, t)-v_{x x}(x, t)+\frac{1}{2} F_{1}(v(x, t))+F_{2}(v(x, t))=g(x, t),
$$

subject to the initial condition

$$
v(x, 0)=f(x)
$$

where $g(x, t)$ is the source term, and $F_{1}(v(x, t))$ and $F_{2}(v(x, t))$ are representing the nonlinear terms, $v_{x}^{2}(x, t)$ and $v^{2}(x, t)$ of the unknown function $v(x, t)$. Many numerical techniques has been used to solve gas dynamic equations such as Decomposition Method (DM) [1], Reduce Differential Transform Method [2], Reconstruction of variational Iteration Method (RVIM) [3], Fourier Transform Adomian Decomposition Method (FTADM) [4], Homotopy Perturbation Method (HPM) [5], Variational Iterative Method (VIM) [6], El-Zaki Transform Homotopy Perturbation Method (ETHPM) [7], and so on.

In this paper, we introduce a new technique called the Natural Decomposition Method (NDM) for solving homogeneous and inhomogeneous gas dynamic equations. The new technique is a combination of the Natural transform Method (NTM) and Adomian Decomposition Method (ADM). It does not require any unnecessary linearization, discretization, transformation or taking some restrictive assumptions and it avoids round off errors. The new technique lead to exact or approximate solution in form of a rapidly convergence series with elegant computational terms. Exact solution of two illustrative examples are successfully fund using the new technique. Hence, the Natural Decomposition Method is a powerful mathematical technique for solving gas dynamic equations and can easily be applied to solve many nonlinear partial differential equations.

The remaining part of this paper is organized as follows: In Section 2, we begin with the Mathematical Preliminaries. In section 3, we present the Analysis of the Natural Decomposition Method. In Section 4, we present some applications of the (NDM) to show its simplicity, effectiveness and accuracy. Finally in Section 5 conclusion and section 6, we gave the references of this paper. 


\section{Mathematical Preliminaries}

In this section, we present some definitions and properties of the Natural transform.

Definition: The Natural transform of the function $f(t) \in A$, for $t \in(0, \infty)$ is defined by $[14,15]$ :

$$
\mathbb{N}^{+}[f(t)]=V(s, u)=\int_{0}^{\infty} e^{-s t} f(u t) d t ; \quad s, u \in(0, \infty),
$$

where $\mathbb{N}^{+}[f(t)]$ is the Natural transformation of the time function $f(t)$, $A=\left\{f(t): \exists M, \tau_{1}, \tau_{2}>0,|f(t)|<M e^{\frac{|t|}{\tau_{j}}}\right.$, where $\left.t \in(-1)^{j} \times[0, \infty)\right\}$, where $j$ is a non-negative integer, and the variables $s$ and $u$ are the Natural transform variables.

Basic theorem of the Natural transform method are given below. See[15, 16, 17]:

Theorem 1 If $V(s, u)$ is the Natural transform and $F(s)$ is the Laplace transform of the function $f(t) \in A$, then $\mathbb{N}^{+}[f(t)]=V(s, u)=\frac{1}{u} \int_{0}^{\infty} e^{-\frac{s t}{u}} f(t) d t=$ $\frac{1}{u} F\left(\frac{s}{u}\right)$.

Theorem 2 If $V(s, u)$ is the Natural transform and $G(u)$ is the Sumudu transform of the function $f(t) \in A$, then $\mathbb{N}^{+}[f(t)]=V(s, u)=\frac{1}{s} \int_{0}^{\infty} e^{-t} f\left(\frac{u t}{s}\right) d t=$ $\frac{1}{s} G\left(\frac{u}{s}\right)$.

Theorem 3 If $\mathbb{N}^{+}[f(t)]=V(s, u)$, then $\mathbb{N}^{+}[f(a t)]=\frac{1}{a} V(s, u)$.

Theorem 4 If $\mathbb{N}^{+}[f(t)]=V(s, u)$, then $\mathbb{N}^{+}\left[f^{\prime}(t)\right]=\frac{s}{u} V(s, u)-\frac{f(0)}{u}$.

Theorem 5 If $\mathbb{N}^{+}[f(t)]=V(s, u)$, then $\mathbb{N}^{+}\left[f^{\prime \prime}(t)\right]=\frac{s^{2}}{u^{2}} V(s, u)-\frac{s}{u^{2}} f(0)-$ $\frac{f^{\prime}(0)}{u}$.

Remark The Natural transform is a linear operator. That is, if $\alpha$ and $\beta$ are non-zero constants, then

$\mathbb{N}^{+}[\alpha f(t) \pm \beta g(t)]=\alpha \mathbb{N}^{+}[f(t)] \pm \beta \mathbb{N}^{+}[g(t)]=\alpha F^{+}(s, u) \pm \beta G^{+}(s, u)$.

Moreover, $F^{+}(s, u)$ and $G^{+}(s, u)$ are the Natural transforms of $f(t)$ and $g(t)$, respectively. 
Table 1. List of some special Natural transforms of some functions. See[15]

\begin{tabular}{|c|c|}
\hline Functional Form & Natural Transform Form \\
\hline 1 & $\frac{1}{s}$ \\
\hline$t$ & $\frac{u}{s^{2}}$ \\
\hline$e^{a t}$ & $\frac{1}{s-a u}$ \\
\hline$\frac{t^{n-1}}{(n-1) !}, n=1,2, \ldots$ & $\frac{u^{n-1}}{s^{n}}$ \\
\hline $\sin (t)$ & $\frac{u}{s^{2}+u^{2}}$ \\
\hline
\end{tabular}

\section{Analysis of the Method}

In this section, we illustrate the basic idea of the Natural Decomposition Method (NDM) for general nonlinear gas dynamic equation of the form (1.1)(1.2):

$$
v_{t}(x, t)-v_{x x}(x, t)+\frac{1}{2} F_{1}(v(x, t))+F_{2}(v(x, t))=g(x, t),
$$

subject to the initial condition

$$
v(x, 0)=f(x)
$$

where $g(x, t)$ is the source term, and $F_{1}(v(x, t))$ and $F_{2}(v(x, t))$ represent the nonlinear terms $v_{x}^{2}(x, t)$ and $v^{2}(x, t)$ of the unknown function $v(x, t)$.

Applying the Natural transform on both sides of Eq. (3.1), we obtain:

$$
\frac{s}{u} V(x, s, u)-\frac{1}{u} v(x, 0)-\mathbb{N}^{+}[v(x, t)]+\mathbb{N}^{+}\left[\frac{1}{2} F_{1}(v(x, t))+F_{2}(v(x, t))\right]=\mathbb{N}[g(x, t)] .
$$

Substituting the given initial condition of Eq. (3.2) into Eq. (3.3), we obtain:

$V(x, s, u)=\frac{1}{s} f(x)+\frac{u}{s} \mathbb{N}^{+}[g(x, t)]+\frac{u}{s} \mathbb{N}^{+}\left[v(x, t)-\frac{1}{2} F_{1}(v(x, t))-F_{2}(v(x, t))\right]$.

Taking the inverse Natural transform of Eq. (3.4), we obtain

$$
v(x, t)=G(x, t)+\mathbb{N}^{-1}\left[\frac{u}{s} \mathbb{N}^{+}\left[v(x, t)-\frac{1}{2} F_{1}(v(x, t))-F_{2}(v(x, t))\right]\right],
$$

where $G(x, t)$ is a term arising from the source terms. 
Now we assume an infinite series solutions for the unknown functions $v(x, t)$ of the form:

$$
v(x, t)=\sum_{n=0}^{\infty} v_{n}(x, t) .
$$

The nonlinear terms $F_{1}(v(x, t))$ and $F_{2}(v(x, t))$ can easily be represented by decomposition series of the form:

$$
F_{1}(v(x, t))=\sum_{n=0}^{\infty} A_{n}(x, t)
$$

and

$$
F_{2}(v(x, t))=\sum_{n=0}^{\infty} B_{n}(x, t),
$$

where $A_{n}$ and $B_{n}$ are Adomian polynomials which represent the nonlinear terms $v_{x}^{2}(x, t)$ and $v^{2}(x, t)$, and can easily be computed using the following formulas:

$$
A_{n}=\frac{1}{n !} \frac{d^{n}}{d x^{n}}\left[F_{1}\left(\sum_{i=0}^{n} \lambda^{i} v_{i}(x, t)\right)\right]_{\lambda=0}
$$

and

$$
B_{n}=\frac{1}{n !} \frac{d^{n}}{d x^{n}}\left[F_{2}\left(\sum_{i=0}^{n} \lambda^{i} v_{i}(x, t)\right)\right]_{\lambda=0},
$$

where $n=0,1,2, \cdots$

Below are some few components of $A_{n}$ and $B_{n}$.

$$
\begin{aligned}
A_{0} & =F_{1}\left(v_{0}\right) \\
& =v_{0 x}^{2} \\
A_{1} & =v_{1 x} F_{1}^{\prime}\left(v_{0}\right) \\
& =2 v_{0 x} v_{1 x}, \\
A_{2} & =v_{2 x} F_{1}^{\prime}\left(v_{0}\right)+\frac{1}{2 !} v_{1 x}^{2} F_{1}^{\prime \prime}\left(v_{0}\right) \\
& =2 v_{0 x} v_{2 x}+v_{1 x}^{2},
\end{aligned}
$$




$$
\begin{aligned}
B_{0} & =F_{2}\left(v_{0}\right) \\
& =v_{0}^{2} \\
B_{1} & =v_{1} F_{2}^{\prime}\left(v_{0}\right) \\
& =2 v_{0} v_{1}, \\
B_{2} & =v_{2} F_{2}^{\prime}\left(v_{0}\right)+\frac{1}{2 !} v_{1}^{2} F_{2}^{\prime \prime}\left(v_{0}\right) \\
& =2 v_{0} v_{2}+v_{1}^{2},
\end{aligned}
$$

and so on.

By substituting Eq. (3.6) and Eq. (3.7) into Eq. (3.5), we obtain:

$$
\sum_{n=0}^{\infty} v_{n}(x, t)=G(x, t)+\mathbb{N}^{-1}\left[\frac{u}{s} \mathbb{N}^{+}\left[\sum_{n=0}^{\infty} v_{n}(x, t)-\frac{1}{2} \sum_{n=0}^{\infty} A_{n}-\sum_{n=0}^{\infty} B_{n}(\beta .] 11\right)\right.
$$

Then by comparing both sides of Eq. (3.9) above, we can easily generate the recursive relation as follows:

$$
\begin{aligned}
& v_{0}(x, t)=G(x, t), \\
& v_{1}(x, t)=\mathbb{N}^{-1}\left[\frac{u}{s} \mathbb{N}^{+}\left[v_{0}(x, t)-\frac{1}{2} A_{0}-B_{0}\right]\right], \\
& v_{2}(x, t)=\mathbb{N}^{-1}\left[\frac{u}{s} \mathbb{N}^{+}\left[v_{1}(x, t)-\frac{1}{2} A_{1}-B_{1}\right]\right], \\
& v_{3}(x, t)=\mathbb{N}^{-1}\left[\frac{u}{s} \mathbb{N}^{+}\left[v_{2}(x, t)-\frac{1}{2} A_{2}-B_{2}\right]\right],
\end{aligned}
$$

and so on.

Thus, the general recursive relation is given by:

$$
v_{n+1}(x, t)=\mathbb{N}^{-1}\left[\frac{u}{s} \mathbb{N}^{+}\left[v(x, t)-\frac{1}{2} A_{n}-B_{n}\right]\right], n \geq 0 .
$$

Hence, the exact or approximate solutions of the unknown function $v(x, t)$ is given by:

$$
v(x, t)=\sum_{n=0}^{\infty} v_{n}(x, t)
$$

\section{Applications}

In this section, we illustrate the applicability of the Natural Decomposition Method to homogeneous and inhomogeneous gas dynamics equations. 
Example 4.1 Consider the homogeneous gas dynamic equation of the form [1-9]:

$$
v_{t}(x, t)+\frac{1}{2}\left(v^{2}(x, t)\right)_{x}=v_{x x}(x, t)-v^{2}(x, t),
$$

subject to the initial condition:

$$
v(x, 0)=e^{-x}
$$

Applying the Natural transform on both sides of Eq. (4.1), we obtain:

$$
\frac{s}{u} V(x, s, u)-\frac{1}{u} v(x, 0)+\frac{1}{2} \mathbb{N}^{+}\left[\left(v^{2}(x, t)\right)_{x}\right]=\mathbb{N}^{+}\left[v(x, t)-v^{2}(x, t)\right] .
$$

Substituting the given initial condition of Eq. (4.2) into Eq. (4.3), we obtain:

$$
V(x, s, u)=\frac{1}{s} e^{-x}+\frac{u}{s}\left[\mathbb{N}^{+}\left[v(x, t)-\frac{1}{2}\left(v^{2}(x, t)\right)_{x}-v^{2}(x, t)\right]\right] .
$$

Then by taking the inverse Natural transform of Eq. (4.4), we have

$$
v(x, t)=e^{-x}+\mathbb{N}^{-1}\left[\frac{u}{s}\left[\mathbb{N}^{+}\left[v(x, t)-\frac{1}{2}\left(v^{2}(x, t)\right)_{x}-v^{2}(x, t)\right]\right]\right] .
$$

We now assume an infinite series solution of the unknown function $v(x, t)$ of the form:

$$
v(x, t)=\sum_{n=0}^{\infty} v_{n}(x, t)
$$

Then by using Eq. (4.6), we can re-write Eq. (4.5) in the form:

$$
\sum_{n=0}^{\infty} v_{n}(x, t)=e^{-x}+\mathbb{N}^{-1}\left[\frac{u}{s}\left[\mathbb{N}^{+}\left[\sum_{n=0}^{\infty} v_{n}(x, t)-\frac{1}{2} \sum_{n=0}^{\infty} A_{n}-\sum_{n=0}^{\infty} B_{n}\right]\right]\right]
$$

where $A_{n}$ and $B_{n}$ are the Adomian polynomial which represent the nonlinear terms $\left(v^{2}\right)_{x}(x, t)$ and $v^{2}(x, t)$ respectively.

By comparing both sides of Eq. (4.7), we can easily generate the recursive relation as follows:

$$
\begin{aligned}
& v_{0}(x, t)=e^{-x} \\
& v_{1}(x, t)=\mathbb{N}^{-1}\left[\frac{u}{s}\left[\mathbb{N}^{+}\left[v_{0}-\frac{1}{2} A_{0}-B_{0}\right]\right]\right] \\
& v_{2}(x, t)=\mathbb{N}^{-1}\left[\frac{u}{s}\left[\mathbb{N}^{+}\left[v_{1}-\frac{1}{2} A_{1}-B_{1}\right]\right]\right] \\
& v_{3}(x, t)=\mathbb{N}^{-1}\left[\frac{u^{2}}{s^{2}}\left[\mathbb{N}^{+}\left[v_{2}(x, t)-\frac{1}{2} A_{2}-B_{2}\right]\right]\right],
\end{aligned}
$$


Therefore, the general recursive relation of the unknown function $v(x, t)$ is given by:

$$
v_{n+1}(x, t)=\mathbb{N}^{-1}\left[\frac{u}{s}\left[\mathbb{N}^{+}\left[v_{n}(x, t)-\frac{1}{2} A_{n}-B_{n}\right]\right]\right], n \geq 0 .
$$

Using the general recursive relation of Eq. (4.8), we can easily compute the remaining components of the unknown function $v(x, t)$ as follows:

$$
\begin{aligned}
v_{1}(x, t) & =\mathbb{N}^{-1}\left[\frac{u}{s}\left[\mathbb{N}^{+}\left[v_{0}(x, t)-\frac{1}{2} A_{0}-B_{0}\right]\right]\right] \\
& =\mathbb{N}^{-1}\left[\frac{u}{s}\left[\mathbb{N}^{+}\left[v_{0}(x, t)-\frac{1}{2}\left(v^{2}\right)_{0 x}-v_{0}^{2}\right]\right]\right] \\
& =\mathbb{N}^{-1}\left[\frac{u}{s}\left[\mathbb{N}^{+}\left[e^{-x}\right]\right]\right] \\
& =e^{-x} \mathbb{N}^{-1}\left[\frac{u}{s}\left[\mathbb{N}^{+}[1]\right]\right] \\
& =e^{-x} \mathbb{N}^{-1}\left[\frac{u}{s^{2}}\right] \\
& =\frac{t}{1 !} e^{-x}
\end{aligned}
$$

$$
\begin{aligned}
v_{2}(x, t) & =\mathbb{N}^{-1}\left[\frac{u}{s}\left[\mathbb{N}^{+}\left[v_{1}(x, t)-\frac{1}{2} A_{1}-B_{1}\right]\right]\right] \\
& =\mathbb{N}^{-1}\left[\frac{u}{s}\left[\mathbb{N}^{+}\left[v_{1}(x, t)-\left(v_{0} v_{1}\right)_{x}-2 v_{0} v_{1}\right]\right]\right] \\
& =\mathbb{N}^{-1}\left[\frac{u}{s}\left[\mathbb{N}^{+}\left[t e^{-x}\right]\right]\right] \\
& =e^{-x} \mathbb{N}^{-1}\left[\frac{u}{s}\left[\mathbb{N}^{+}[t]\right]\right] \\
& =e^{-x} \mathbb{N}^{-1}\left[\frac{u^{2}}{s^{3}}\right] \\
& =\frac{t^{2}}{2 !} e^{-x}
\end{aligned}
$$




$$
\begin{aligned}
v_{3}(x, t) & =\mathbb{N}^{-1}\left[\frac{u}{s}\left[\mathbb{N}^{+}\left[v_{2}(x, t)-\frac{1}{2} A_{2}-B_{2}\right]\right]\right] \\
& =\mathbb{N}^{-1}\left[\frac{u}{s}\left[\mathbb{N}^{+}\left[v_{2}(x, t)-\left(v_{0} v_{2}\right)_{x}-\frac{1}{2} v_{1 x}-2 v_{0} v_{2}-v_{1}^{2}\right]\right]\right] \\
& =\mathbb{N}^{-1}\left[\frac{u}{s}\left[\mathbb{N}^{+}\left[\frac{t^{2}}{2 !} e^{-x}\right]\right]\right] \\
& =\frac{1}{2 !} e^{-x} \mathbb{N}^{-1}\left[\frac{u}{s}\left[\mathbb{N}^{+}\left[t^{2}\right]\right]\right] \\
& =\frac{1}{2 !} e^{-x} \mathbb{N}^{-1}\left[\frac{2 u^{3}}{s^{4}}\right] \\
& =\frac{t^{3}}{3 !} e^{-x}
\end{aligned}
$$

and so on.

Hence, the approximate series solution of the unknown function $v(x, t)$ is given by:

$$
\begin{aligned}
v(x, t) & =\sum_{n=0}^{\infty} v_{n}(x, t) \\
& =v_{0}(x, t)+v_{1}(x, t)+v_{2}(x, t)+v_{3}(x, t)+\cdots \\
& =e^{-x}+\frac{t}{1 !} e^{-x}+\frac{t^{2}}{2 !} e^{-x}+\frac{t^{3}}{3 !} e^{-x}+\cdots \\
& =e^{t-x}
\end{aligned}
$$

Therefore, the exact solution of the gas dynamic equation (4.1)-(4.2) is given by:

$$
v(x, t)=e^{t-x}
$$

The exact solution is in closed agreement with the result obtained by (DM) [2], (RDTM) [2], (RVIM) [3], (FTADM) [4], (HPM) [5], and (VIM) [6]

Example 4.2 Consider the inhomogeneous gas dynamics equation of the form $[3,7]$ :

$$
v_{t}(x, t)+\frac{1}{2}\left(v^{2}(x, t)\right)_{x}=v_{x x}(x, t)-v^{2}(x, t)-e^{t-x},
$$

subject to the initial condition:

$$
v(x, 0)=1-e^{-x} .
$$


Applying the Natural transform on both sides of Eq. (4.9), we obtain:

$$
\frac{s}{u} V(x, s, u)-\frac{1}{u} v(x, 0)+\frac{1}{2} \mathbb{N}^{+}\left[\left(v^{2}(x, t)\right)_{x}\right]=\frac{e^{-x}}{s-u}+\mathbb{N}^{+}\left[v(x, t)-v^{2}(x, t)\right] .
$$

Substituting the given initial condition of Eq. (4.10) into Eq. (4.11), we obtain:

$$
V(x, s, u)=\frac{1-e^{-x}}{s}+\frac{u e^{-x}}{s(s-u)}+\frac{u}{s}\left[\mathbb{N}^{+}\left[v(x, t)-\frac{1}{2}\left(v^{2}(x, t)\right)_{x}-v^{2}(x, t)\right]\right] .
$$

Then by taking the inverse Natural transform of Eq. (4.12), we have

$$
v(x, t)=1-e^{t-x}+\mathbb{N}^{-1}\left[\frac{u}{s}\left[\mathbb{N}^{+}\left[v(x, t)-\frac{1}{2}\left(v^{2}(x, t)\right)_{x}-v^{2}(x, t)\right]\right]\right],
$$

since, $\mathbb{N}^{-1}\left[\frac{u e^{-x}}{s(s-u)}\right]=e^{-x} \mathbb{N}^{-1}\left[\frac{u}{s(s-u)}\right]=e^{-x}\left(e^{t}-1\right)$.

We now assume an infinite series solution of the unknown function $v(x, t)$ of the form:

$$
v(x, t)=\sum_{n=0}^{\infty} v_{n}(x, t)
$$

Then by using Eq. (4.14), we can re-write Eq. (4.13) in the form:

$$
\sum_{n=0}^{\infty} v_{n}(x, t)=1-e^{t-x}+\mathbb{N}^{-1}\left[\frac{u}{s}\left[\mathbb{N}^{+}\left[\sum_{n=0}^{\infty} v_{n}(x, t)-\frac{1}{2} \sum_{n=0}^{\infty} A_{n}-\sum_{n=0}^{\infty} B_{n}\right]\right]\right]
$$

where $A_{n}$ and $B_{n}$ are the Adomian polynomial which represent the nonlinear terms $\left(v^{2}\right)_{x}(x, t)$ and $v^{2}(x, t)$ respectively.

By comparing both sides of Eq. (4.15), we can easily generate the recursive relation as follows:

$$
\begin{aligned}
& v_{0}(x, t)=1-e^{t-x} \\
& v_{1}(x, t)=\mathbb{N}^{-1}\left[\frac{u}{s}\left[\mathbb{N}^{+}\left[v_{0}-\frac{1}{2} A_{0}-B_{0}\right]\right]\right] \\
& v_{2}(x, t)=\mathbb{N}^{-1}\left[\frac{u}{s}\left[\mathbb{N}^{+}\left[v_{1}-\frac{1}{2} A_{1}-B_{1}\right]\right]\right] \\
& v_{3}(x, t)=\mathbb{N}^{-1}\left[\frac{u^{2}}{s^{2}}\left[\mathbb{N}^{+}\left[v_{2}(x, t)-\frac{1}{2} A_{2}-B_{2}\right]\right]\right],
\end{aligned}
$$


Thus, the general recursive relation of the unknown function $v(x, t)$ is given by:

$$
v_{n+1}(x, t)=\mathbb{N}^{-1}\left[\frac{u}{s}\left[\mathbb{N}^{+}\left[v_{n}(x, t)-\frac{1}{2} A_{n}-B_{n}\right]\right]\right], n \geq 0 .
$$

$B y$ using the general recursive relation of Eq. (4.16), we can easily compute the remaining components of the unknown function $v(x, t)$ as follows:

$$
\begin{aligned}
v_{1}(x, t) & =\mathbb{N}^{-1}\left[\frac{u}{s}\left[\mathbb{N}^{+}\left[v_{0}(x, t)-\frac{1}{2} A_{0}-B_{0}\right]\right]\right] \\
& =\mathbb{N}^{-1}\left[\frac{u}{s}\left[\mathbb{N}^{+}\left[v_{0}(x, t)-\frac{1}{2}\left(v^{2}\right)_{0 x}-v_{0}^{2}\right]\right]\right] \\
& =0 .
\end{aligned}
$$

Thus,

$$
v_{n+1}(x, t)=0, \quad n \geq 0 .
$$

Hence, the approximate series solution of the unknown function $v(x, t)$ is given by:

$$
\begin{aligned}
v(x, t) & =\sum_{n=0}^{\infty} v_{n}(x, t) \\
& =v_{0}(x, t)+v_{1}(x, t)+v_{2}(x, t)+v_{3}(x, t)+\cdots \\
& =1-e^{t-x}+0+0+0+\cdots \\
& =1-e^{t-x}
\end{aligned}
$$

Therefore, the exact solution of the inhomogeneous gas dynamics equation (4.9)-(4.10) is given by:

$$
v(x, t)=1-e^{t-x}
$$

The exact solution is in closed agreement with the result obtained by (RVIM) [3] and (ETHPM) [7]

\section{Conclusion}

In this paper, we introduce an efficient technique called the Natural Decomposition Method for solving gas dynamics equation without using any transformation, linearization, discritization or taking some restrictive assumptions. 
The new technique provides an elegant series solution which converge very rapidly with reduced computational size, and avoids round off errors. The exact solution obtained by the new technique is in excellent agreement with the results of the existing methods. Thus, the proposed technique is a powerful, reliable and efficient mathematical tool for solving nonlinear partial differential equations.

\section{Acknowledgement}

The authors are very grateful to the editor and the anonymous referees for their comments and suggestions which have led to improvement of the paper.

\section{References}

[1] D.J. Evans, H. Bulut, A new approach to the gas dynamics equations: An application of the decomposition method, Appl. Comp. Math., 79 (2002), $817-822$.

[2] Yildiray Keskin, Galip Oturanc Application of Reduce Differential Transformation Method for solving Gas Dynamics Equation, Int. Contemp. Sciences, 5(22) (2010), 1091-1096.

[3] A. Nikkar, A new approach for solving gas dynamic equation, Acta Technica Corviniensis Bulletin of Engineering, 4 (2012), 2067-3809.

[4] Masoud Ramezanpour, Nadar Montazerin, Payam Izady and Ahmed Doosthoseini, A new algorithm to solve the gas dynamics equation: An application of the Fourier Transform Adomian Decomposition Method, Applied Mathematical Sciences, 7(86) (2013), 4281-4286.

[5] H. Jafari, M. Zabihi, M. saidy, Application of homotopy perturbation method for solving gas dynamics equation, Applied Mathematical Sciences, 2 (2008), 2393-2396.

[6] H. Jafari, S. Hosseinzadehb and E. salehpoor, A new approach to gas dynamics equation: Application of Variational Iteration Method, Applied Mathematical Sciences, 2(48) (2008), 2397-2400.

[7] Prem Kiran G. Bhadane, V.H. Pradhan, El-Zaki Transform Homotopy Perturbation Method for solving gas dynamics equation, International Journal of Reseach in Engr. and Tech., 2 (2013), eISSN:2319-1163, pISSN:2321-7308. 
[8] Hossein Aminikhah, Ali Jamalian, Numerical Approximation for nonlinear gas dynamics equation,, International Journal of Partial Differerential Equations, (2013),Article ID 8467497.

[9] M. Matinfar, M. Saeidy, M. Mahdari and M. Rezael, Variational Iteration Method for exact solution of gas dynamics equation using He's Polynomials, Bulletin of Mathematical Analysis and Applications, 3(3) (2011), $50-55$.

[10] . Adomian, Solving frontier problems of physics: the decomposition method, Kluwer Acad. Publ (1994).

[11] G. Adomian, A new approach to nonlinear partial differential equations, J. Math. Anal. Appl., 102 (1984), 420-434.

[12] El-sayed, The decomposition method for studying the Klein-Gordon Equation, Chaos, Soliton and Fractals , 18 (2003), 1025-1030.

[13] J. Biarzar, E. Babolian, R. Islam, An alternative algorithm for computing Adomian polynomial in special cases, Applied Mathematics and Computation, 138 (2003), 523-529.

[14] Wazwaz A., The modified decomposition method for analytic treatment of differential equations, Applied Mathematics and Computations, 173 (2006), 165-176.

[15] Fethi Bin. Muhammed Belgacem, R. Silambarasan, Theory of Natural Transform, Mathematical Engineering, Science and Aerospace., 3 (1) (2012), 100-124.

[16] F.B.M. Belgacem, R. Silambarasan, Maxwell's equations by means of the natural transform, Mathematics in Engineering, Science and aerospace, 3 (2012), 313-323.

[17] Z.H. Khan, W.A. Khan, N-transform properties and applications, NUST Jour. of Engg. Sciences, 1 (2008), 127-133.

[18] Hussain, M. Khan, A variationa iteration method for solving linear and nonlinear Klein-Gordon equation, Applied Mathematical Science, 4 (2010), 1931-1940.

[19] F.B.M. Belgacem, R. Silambarasan, Theoretical investigations of the natural transform, Progress In Electromagnetics Research Symposium Proceedings, Suzhou, China, Sept. 12 (16) (2011). 
[20] F.B.M. Belgacem, R. Silambarasan, Maxwell's equations solutions through the natural transform, Mathematics in Engineering, Science and Aerospace 3 (3) (2012), 313-323.

[21] M.G.M. Hussain, F.B.M. Belgacem, Transient solutions of Maxwell's equations based on Sumudu transform, Progress in Electromagnetics Research, 74 (2007), 273-289.

[22] M.R. Spiegel, Theory and Problems of Laplace Transforms, Schaums Outline Series, McGraw-Hill, New York (1965).

[23] S.T. Khuri, A Laplace decomposition algorithm applied to a class of nonlinear partial differential equations, Journal of Applied Mathematics, 1 (2001), 141-155.

[24] Belgacem, F. B. M., Karaballi, A. A., Kalla, S. L.; Analytical investigations of the Sumudu transform and applications to integral production equations, Mathematical Problems in Engineering, 3 (2003), 103-118.

[25] Belgacem, F. B. M., Karaballi, A. A., Sumudu transform fundamental properties, investigations and applications, Journal of Applied Mathematics and Stochastic Analysis, 40 (2006), 1-23.

\section{Received: May 15, 2014}

